\title{
AN ALTERNATIVE PROOF OF DIESTEL'S THEOREM
}

\author{
by M. ALI KHAN
}

(Received 28 July, 1982)

Introduction. We show that Diestel's theorem on weak compactness of subsets of $L_{1}(\mu, X)$ can be derived as a simple corollary of James's theorem. It is a pleasure to acknowledge several stimulating conserversations with Dave Emmons and the remarks of an anonymous referee. Errors are, of course, solely mine.

Let $(T, \mathscr{T}, \mu)$ be a finite measure space and $X$ a Banach space. Denote by $L_{1}(\mu, X)$ the Banach space of (equivalence classes of) $\mu$-strongly measurable $X$-valued Bochner integrable functions $f: T \rightarrow X$ normed by $\|f\|_{1}=\int_{T}\|f(t)\| d \mu(t)$. In [3] Diestel has proved through the use of the factorization method in [2] the following result.

THEOREM. Let $K$ be a weakly compact convex subset of $X$ and

$$
\tilde{K}=\left\{f \in L_{1}(\mu, X): f(t) \in K \text { for almost all } t \text { in } T\right\} ;
$$

then $\tilde{K}$ is weakly compact in $L_{1}(\mu, X)$.

In this note, we offer an alternative proof of Diestel's theorem which relies instead on James's theorem [5] and on Brooks's extension of the classical Vitali's theorem [1].

Before presenting our proof, we recall that $V_{\infty}\left(\mu, X^{*}\right)$, where $X^{*}$ is the continuous dual of $X$, is isometrically isomorphic to $L_{1}(\mu, X)^{*}$ with the correspondence between $F \in V_{\infty}\left(\mu, X^{*}\right)$ and $\phi \in L_{1}(\mu, X)^{*}$ given by $\phi(f)=\int f d F$. (For an explanation and properties of $V_{\infty}\left(\mu, X^{*}\right)$ see [3] and his references.)

Proof. Pick an arbitrary $\phi \in\left(L_{1}(\mu, X)\right)^{*}$. If we can show that $\phi$ attains its supremum on $\tilde{K}$, James's theorem [5, Theorem 5] assures us that $\tilde{K}$ is relatively weakly compact. Since $\tilde{K}$ is convex and closed (hence by Mazur's theorem weakly closed) in $L_{1}(\mu, X)$, the proof is then finished.

Let $F \in V_{\infty}\left(\mu, X^{*}\right)$ correspond to $\phi$. Towards showing that $\phi$ attains its supremum on $\tilde{K}$, select a pairwise disjoint sequence of elements $T_{i} \in \mathscr{T}$ such that each $T_{i}$ has positive measure and $\bigcup_{i=1}^{\infty} T_{i}=T$. Consider the finite partition $\pi_{n}=\left\{T_{1}, T_{2}, \ldots, T_{n-1}, \bigcup_{i=n}^{\infty} T_{i}\right\}$ in which $T_{n}$ will denote $\bigcup_{i=n}^{\infty} T_{i}$. Let $\pi=\left\{\pi_{n}\right\}_{n \geq 1}$. It is clear that for all integers $n, \pi_{n+1}$ is a refinement of $\pi_{n}$.

For any partition $\pi_{n}$ construct the function $f_{n} \in L_{1}(\mu, X)$ such that

$$
f_{n}(t)=x_{i} \text { for all } t \in T_{i}, \quad(i=1, \ldots, n),
$$

where $x_{i}$ is characterized by the equality $\left\langle x_{i}, F\left(T_{i}\right)\right\rangle=\sup _{y \in K}\left\langle y, F\left(T_{i}\right)\right\rangle$. Since $K$ is nonempty and weakly compact, certainly $x_{i} \in K$ for all $i$. We are now going to take a suitable limit of a subsequence of these functions.

Glasgow Math. J. 25 (1984) 45-46. 
By our construction, for any $t$ in $T_{1}$, we can let $f_{n}(t)=f_{n}\left(T_{1}\right)$, where $f_{n}\left(T_{1}\right) \in K$ for all $n$. Since $K$ is weakly compact, the Eberlein-Šmulian Theorem guarantees a subsequence $f_{n}^{(1)}\left(T_{1}\right)$ which converges weakly to an element, say $f\left(T_{1}\right)$, in $K$. The procedure is now clear. We can now manufacture a function $f: T \rightarrow K$ such that $f$ is the almost everywhere limit of $f_{n}^{(n)}$, where for any $i=1, \ldots, n-1, f_{n}^{(i+1)}$ is a subsequence of $f_{n}^{(i)}$ such that for all $n, f_{n}^{(i)}(t)=f_{n}^{(i)}\left(T_{i+1}\right)$ for all $t$ in $T_{i+1}$ and $f_{n}^{(i+1)}\left(T_{i+1}\right)$ converges weakly to an element, say $f\left(T_{i+1}\right)$, in $K$.

Since $K$ is weakly compact, for all $x \in K$ there exists $M>0$ such that $\|x\| \leq M$. Using this fact it is now easy to show that the sequence $f_{n}^{(n)}$ is bounded and uniformly integrable. We can therefore apply Brooks's extension [1. Theorem 3] of Vitali's convergence theorem to claim that $f \in L_{1}(\mu, X)$ and hence $f \in \tilde{K}$ and that $\left\|f-f_{n}^{(n)}\right\|_{1} \rightarrow 0$. Then, certainly $\int f_{n}^{(n)} d F \rightarrow \int f d F$.

We now claim that $\phi$ attains its supremum on $\tilde{K}$ at $f$. Suppose not; that is there exists $z \in \tilde{K}$ such that

$$
\phi(z)=\int_{T} z d F>\int_{T} f d F=\phi(f) .
$$

For each partition $\pi$ in $\Pi$, define the linear operator $E_{\pi}: L_{1}(\mu, X) \rightarrow L_{1}(\mu, X)$ by

$$
E_{\pi}(z)=\sum_{T_{i} \in \pi}\left[\frac{1}{\mu\left(T_{i}\right)} \int_{T_{i}} z(t) d \mu(t)\right] \chi_{T_{i}},
$$

where $\chi_{\mathrm{A}}$ is the characteristic function of $A$ and the $0 / 0=0$ convention is in force. By the mean value theorem for the Bochner integral [4, Corollary 8, p. 48] and the convexity of $K$, certainly $\frac{1}{\mu\left(T_{i}\right)} \int_{T_{i}} z(t) d \mu(t) \in K$. Thus $E_{\pi}(z) \in \tilde{K}$. We can now apply Lemma 1 in $[3$, p. $67]$ to assert that $\left\|E_{\pi_{n}}(z)-z\right\|_{1} \rightarrow 0$. Then certainly $\int_{T} E_{\pi_{n}}(z) d F \rightarrow \int_{T} z d F$.

However by construction,

$$
\int_{T} E_{\pi_{n}}(z) d F \leq \int_{T} f_{n}^{(n)} d F .
$$

By taking limits on both sides, we obtain our sought-after contradiction to (1).

\section{REFERENCES}

1. J. K. Brooks, Equicontinuous sets of measures and applications to Vitali's integral convergence theorem and control measures, Advances in Math. 10 (1973), 165-171.

2. W. J. Davis, T. Figiel, W. B. Johnson and A. Pelczynski, Factoring weakly compact operators, J. Functional Analysis 17 (1974), 311-327.

3. J. Diestel, Remarks on weak compactness in $L_{1}(\mu, X)$, Glasgow Math. J. 18 (1977), 87-91.

4. J. Diestel and J. J. Uhl, Jr., Vector measures, American Mathematical Society (Providence, Rhode Island, 1977).

5. R. C. James, Weakly compact sets, Trans. Amer. Math. Soc. 113 (1964), 129-140.

The Johns Hopkins UnIVERSTTY

BALTIMORE

MARYLAND 21218

U.S.A. 